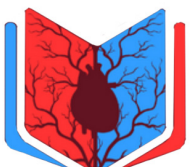

Published By : IVAA

the Indonesian Vascular Access Association

\title{
Anaesthesia management in Chronic Kidney Disease (CKD) patient undergo repair pseudoaneurysms surgery: a case report
}

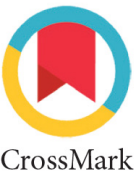

CrossMark
'Resident of Department of Anesthesia and Critical Care Medicine, Faculty of Medicine, Universitas Udayana, Sanglah General Hospital, Bali, Indonesia ${ }^{2}$ Instructor of Department of Anesthesia and Critical Care Medicine, Faculty of Medicine, Universitas Udayana, Sanglah General Hospital, Bali, Indonesia ${ }^{3}$ Associate Professor, Department of Anesthesia and Critical Care Medicine, Faculty of Medicine, Universitas Udayana, Sanglah General Hospital, Bali, Indonesia

\section{*Correspondening to:}

Tan Andi; Resident of Department of Anesthesia and Critical Care Medicine, Faculty of Medicine, Universitas Udayana, Sanglah General Hospital, Bali, Indonesia; tndbius2018@gmail.com

Received:2021-04-26

Accepted: 2021-12-02

Published: 2021-12-28
Tan Andi ${ }^{*}$, Tjahya Aryasa ${ }^{2}$, Tjokorda Gde Agung Senapathi ${ }^{3}$

\section{ABSTRACT}

Introduction: Chronic kidney disease (CKD) is marked by the presence of kidney damage (usually defined as estimated GFR $<60 \mathrm{~mL} / \mathrm{min} / 1.73 \mathrm{m2}$ ) for 3 or more months, and it may be caused by a multitude of disease processes. Management of patients with CKD includes aggressive treatment of the underlying cause, pharmacologic therapy to delay disease progression and prevent complications, and preparation for hemodialysis as ESRD ensues.

Case presentation: In this case report, a 48-year-old man with a pseudoaneurysms due to the insertion of a vascular access for hemodialysis, undergo repair pseudoaneurysms surgery under general anesthesia, at the Sanglah General Hospital, October 2020. The patient came with fully awareness, blood pressure $145 / 95 \mathrm{mmHg}$, heart rate 85 times per minute regular and oxygen saturation $97 \%$ with room air.

Conclusion: General anesthesia in patients with CKD requires an understanding of the pathologic changes that accompany renal disease, co-existing medical conditions, and the impact of reduced renal function on drug pharmacokinetics.

Keywords: Chronic kidney disease, general anesthesia, hemodialysis, pseudoaneurysms.

Cite This Article: Andi, T., Aryasa, T., Senapathi, T.G.A. 2021. Anaesthesia management in Chronic Kidney Disease (CKD) patient undergo repair pseudoaneurysms surgery: a case report. Journal of Indonesia Vascular Access 1(2): 41-45. D0I : 10.51559/jinava.v1i2.13

\section{INTRODUCTION}

Chronic kidney disease (CKD) is marked by the presence of kidney damage (usually defined as estimated GFR $<60 \mathrm{~mL} /$ $\mathrm{min} / 1.73 \mathrm{~m}^{2}$ ) for 3 or more months, and it may be caused by a multitude of disease processes. ${ }^{1-3}$ In most patients, regardless of the cause, a decrease in GFR to less than $25 \mathrm{~mL} / \mathrm{min}$ results in end-stage renal disease (ESRD) requiring dialysis or transplantation. ${ }^{1,3,4}$ Patients with CKD generally require vascular access for dialysis under local or regional anesthesia.

Hemodialysis (HD) is a treatment for patients with kidney problems. HD process uses a special machine to process the patient's blood through a filter, called a dialyzer, and to undergo the treatment process requires HD vascular access., This access serves as a pathway for removing and returning the patient's blood during the HD procedure. There are several accesses that can be used, including Arteriovenous (AV) shunt or fistula (AVF) and AV Graft (AVG) as permanent access, while HD catheters or commonly called double lumen catheters (CDL) are temporary access.

The use of graft in dialysis can also cause repetitive trauma resulting in the weakening of the vascular wall which leads to pseudoaneurysm, especially in AV grafts. A pseudoaneurysm is defined as an incomplete aneurysm due to disruption of the vessel wall or anastomosis site between the graft and blood vessels, consisting of blood or pulsating hematoma covered with tissue. ${ }^{6-9}$ The common cause of a pseudoaneurysm is a penetrating wound that pierces the three layers of the artery wall sideways which can lead to an enlarged pseudoaneurysm that compresses the surrounding tissue and even ruptures. ${ }^{8,10}$

Before an anesthesia procedure is performed, it is necessary to carry out a thorough evaluation to determine that the patient is in optimal medical condition. The management of anesthesia given to
CKD patients requires an understanding of the pathological changes associated with kidney disease, and also an assessment of whether the kidney disease that occurs requires $\mathrm{HD}$, drugs that can reduce kidney function, also needs to be assessed whether the kidney disease is stable, is improving, or deterioration..$^{2-4,8}$ Therefore, this study will discuss and explain the anesthesia management of chronic kidney disease $(\mathrm{CKD})$ patient undergoing repair pseudoaneurysms surgery using the reported case of a patient.

\section{CASE DESCRIPTION}

\section{History}

The patient is a male, 48 years old, from West Nusa Tenggara, admitted to the hospital on $4^{\text {th }}$ October 2020 with a diagnosis of Dextra Jugular Vein Pseudoaneurysm and planned to undergo repair pseudoaneurysms. The patient arrives in a conscious state with a chief complaint of swelling accompanied by 
continuous pain in the right side of the neck, since May 2020 which did not decrease with a change in position. The complaint was initially recognized by the patient after the patient's CDL for HD was released and then repaired at the West Nusa Tenggara Hospital. According to the patient, the CDL re-insertion experienced difficulties and was revised several times. The swelling, which was originally only the size of a marble, has gotten bigger to the size of an adult's fist until now. Complaints are accompanied by snoring sleep, hoarseness of voice where the patient is more comfortable sleeping on his side facing the left side. The patient also complained of weakness in slowly moving his right arm so that he could not move again. Swelling without fever, pus, or blood. The appetite to eat and drink is said to be still good.

Current therapy is routine HD twice a week (last HD one day before surgery without heparin administration), amlodipine $10 \mathrm{mg}$ every 24 hours, candesartan $32 \mathrm{mg}$ every 24 hours, bisoprolol $5 \mathrm{mg}$ every 24 hours, cefoperazone lgram every 12 hours, azithromycin $500 \mathrm{mg}$ every 24 hours. The patient has a history of CKD stage $\mathrm{V}$ on HD regular since 2005 twice a week, history of hypertension since 2010 was not controlled. History of allergies, asthma, type 2 Diabetes Mellitus, and other heart diseases were denied. History of surgery previously was also denied.

\section{Physical examination}

Based on physical examination, the patient weight is $85 \mathrm{~kg}$ with a body height of $178 \mathrm{~cm}$ and BMI: $26.82 \mathrm{~kg} / \mathrm{m} 2$. The temperature axilla was $36.9^{\circ} \mathrm{C}$, with NRS silent $2 / 10$ and NRS moves $4 / 10$. Based on central nervous system examination, the patient was compos mentis. By respiration, examination found respiration rate $16 \mathrm{x} /$ minute; symmetrical; vesicular, rhonchi and wheezing absent, oxygen saturation 92-97\% room air. From cardiovascular examination found blood pressure 145/95 mmHg, pulse $89 \mathrm{x} / \mathrm{min}$; regular; murmur (-). From gastrointestinal examination found no distension, normal bowel sounds. From urogenital examination found spontaneous urination, urine output $\pm 500-1000 \mathrm{ml}$ per day. Also from musculoskeletal examination found neck flexion deflection is normal, Mallampati II, Superior motor 0000/5555, inferior $5555 / 5555$

\section{Supporting Examination}

Complete blood count on $4^{\text {th }}$ October 2020 found WBC $8.66 \times 10^{3} / \mu \mathrm{L}$ (4.1-11); HGB $8.69 \mathrm{~g} / \mathrm{dL}$ (12-16); HCT 27.4\% (36-46); PLT $215.10 \times 10^{3} \mu \mathrm{L}$ (140-440). Coagulation screening on $4^{\text {th }}$ October 2020 found PT 14.6 (10.8-14.4) seconds; aPTT 34.3 (2436) seconds; INR 1.04. Clinical Chemistry laboratory examination on $12^{\text {th }}$ October
2020 found BUN $53.00 \mathrm{mg} / \mathrm{dL}$ (8-23); SC $8.71 \mathrm{mg} / \mathrm{dL}(0.5-0.9)$; SGOT $18.6 \mathrm{U} / \mathrm{L}$ (1133); SGPT 76.3 U/L (11-50); BS 85 mmol/L (70-140); albumin 4.0 (3,4-4,8); Na 131 $\mathrm{mmol} / \mathrm{L}$ (136-145); K $4.06 \mathrm{mmol} / \mathrm{L}$ (3.55.1); CL 105mmol/L (96-107). Blood Gas Analysis on $4^{\text {th }}$ October 2020, with oxygen room air, Body Temperatur $36.7^{\prime} \mathrm{C}$ found $\mathrm{pH}$ 7.34; $\mathrm{pCO} 2$ 36.9; $\mathrm{pO} 2$ 121.9; $\mathrm{HCO} 3$ 19.40; BE -6.4; SO2c 98.2. Covid-19 PCR Swabs on $10^{\text {th }}$ October 2020 was Negative. Chest Xray on $9^{\text {th }}$ September 2020 found cardiomegaly CTR 55\%, Pneumonia. Lastly, ECG on $4^{\text {th }}$ October 2020 found
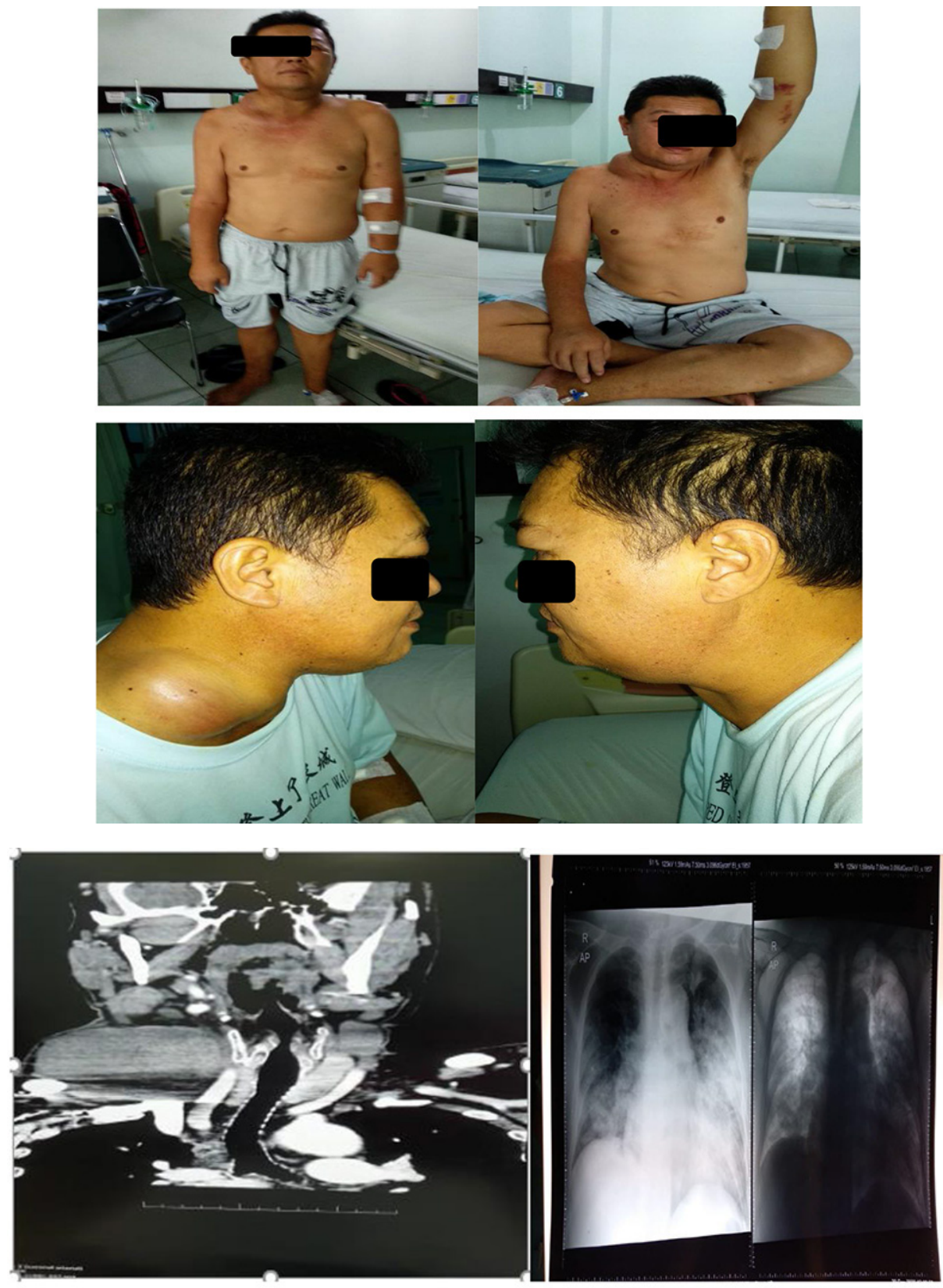

Figure 1. A radiological image of the patient found a mass on the right neck 
normal sinus rhythm, heart rate 75 beats per minute, normal axis, ST-T changes absent

\section{Anesthesia Management}

The patient's preoperative preparation has been started since the patient agreed to the anesthesia procedure. Preparations are carried out in the ward, anesthesia preparation room, and operating room. A complete and good informed consent to the patient and family about the general anesthesia to be performed up to the worst risk of complications due to the anesthesia procedure. In the ward, ensure that intravenous access is available and that crystalloid fluids are installed properly before the patient starts fasting for 8 hours.

When in the preparation room, recheck the patient to make sure all intravenous access is working properly and not phlebitis, then give midazolam 1 mg IV for premedication. The patient is brought into the operating room with a patent upper airway and pulse oximetry attached. Install all monitoring devices such as manual blood pressure, ECG electrodes, oxygen saturation, and oxygen source with facemask before induction begins after the patient is in the operating room. Arterial line access was installed for monitoring blood pressure during surgery. The patient was then induced with propofol titration IV until the patient was hypnotized and facilitated intubation with fentanyl $150 \mathrm{mcg}$ IV; atracurium $50 \mathrm{mg}$ IV; intratracheal lidocaine $120 \mathrm{mg}$. Perform laryngoscopy properly to get a clear view of the vocal cord and not injure the soft tissue around the oral cavity. Place the 7.5 cuffed endotracheal (ET) tube and fix it with adhesive after evaluating the position of the ET tube by auscultating both lungs. Then put the packing properly and label it so you don't forget it. Maintenance of anesthesia was maintained with $\mathrm{O} 250 \%$; Compressed air; Sevoflurane; Propofol continuous $50-150 \mathrm{mcg} / \mathrm{Kg}$ body weight/ minute; fentanyl intermittent; atracurium intermittent. Hemodynamic during surgery with blood pressure fluctuations 50-120 / 30-85 mmHg, heart rate 95$145 \mathrm{x} /$ minute, respiratory rate $20-25 \mathrm{x} /$ minute, oxygen saturation $98-100 \%$. The surgery was performed for 3 hours with a fluid balance of $3000 \mathrm{ml}$ of crystalloid fluids, $1500 \mathrm{ml}$ of colloids, $500 \mathrm{ml}$ of PRC, and with estimated bleeding of $3500 \mathrm{ml}$ and $500 \mathrm{ml}$ of urine output. Then the postoperative patient was transferred to an intensive care unit with a ventilator for postoperative management and analgesic management with fentanyl $15 \mathrm{mcg} / \mathrm{hour}$ via a syringe pump, paracetamol $1000 \mathrm{mg}$ every 8 hours IV.

\section{DISCUSSION}

CKD is marked by the presence of kidney damage (usually defined as estimated GFR $<60 \mathrm{~mL} / \mathrm{min} / 1.73 \mathrm{~m}^{2}$ ) for 3 or more months, and it may be caused by a multitude of disease processes. The most common causes of CKD are hypertensive nephrosclerosis, diabetic nephropathy, chronic glomerulonephritis, and polycystic kidney disease. The clinical manifestations of CKD reflect the inability of the kidneys to excrete nitrogenous waste products, regulate fluid and electrolyte balance, and secrete hormones. ${ }^{1-3,5}$

Understanding the pathologic changes that follow a renal illness, co-existing medical disorders, and the influence of diminished renal function on medication pharmacokinetics are all important aspects of anesthesia care in patients with CKD. Renal function, underlying pathologic processes, and concomitant diseases are all considered in the preoperative evaluation of patients with CKD. Patients with preexisting renal dysfunction must be identified, as well as those who are at high risk of developing perioperative renal failure. ${ }^{1,3,4,8}$

End-stage renal disease (ESRD) is defined as a decline in GFR of less than $25 \mathrm{~mL} / \mathrm{min}$ that requires dialysis or transplantation in the majority of patients, regardless of the reason. This patient has the end-stage renal disease as a result of high blood pressure. Before undergoing elective surgery, be sure your blood pressure is under control. Antihypertensive medicine is routinely continued; however, on the day of surgery, ACE inhibitors and ARBs are frequently withheld to reduce the risk of intraoperative hypotension. ${ }^{5,6}$

In CKD patients, anemia is often found caused by impaired erythropoietin production. ${ }^{5,6}$ In this patient also found anemia (hemoglobin $10.3 \mathrm{~g} / \mathrm{dL}$ ). Patients should undergo adequate $\mathrm{HD}$ within 24 hours of elective surgery to minimize the likelihood of volume overload, hyperkalemia, and uremic bleeding. ${ }^{1,3,4,8}$ Heparin may be avoided or limited during preoperative HD depending on the intended surgery. Patients who are on peritoneal dialysis who are having abdominal surgery are usually shifted to HD right after the surgery. On the day of surgery, the serum potassium content should not exceed $5.5 \mathrm{mEq} /$ L. ${ }^{1,3,4,8}$ This patient had undergone HD one day preoperatively and obtained a serum potassium concentration of $3.8 \mathrm{mEq} / \mathrm{L}$. For the administration of premedication according to the patient's condition, if the patient has complaints of nausea and vomiting or gastrointestinal bleeding, $\mathrm{H} 2$ blockers can be given. In CKD patients, the dose of anesthetic drugs given must be adjusted to their safety. ${ }^{3,8}$

Solutes diffuse over a semipermeable membrane between the blood and the dialysis solution in HD. As a result, metabolic waste products and excess fluid volume are removed, and bodily buffers are replenished. ${ }^{5,6}$ Blood is heparinized and runs through a plastic dialyzer during the procedure. The dialysate, dialysis membrane type, and solute clearance are the most essential variables that can be changed. A normal dialysis session lasts 3 to 4 hours and leads to a BUN reduction of 65 to 70 percent. Patients on HD have a nearly $25 \%$ yearly mortality rate, which is mostly due to cardiovascular disease or infection. ${ }^{1}$ A surgically created vascular access using a central venous catheter (CVC) is necessary for effective hemodialysis. CVC is indicated in addition to monitoring central venous pressure, administering fluids to treat hypovolemia and shock, parenteral nutrition, venous access for patients whose peripheral venous access is difficult to obtain, and can be used as access vascular HD. ${ }^{5-7,10}$ Native arteriovenous Fistulas (e.g., a cephalic vein anastomosed to the radial artery) are better than polytetrafluoroethylene grafts as vascular access sites because they last longer and have a lower risk of thrombosis and infection. ${ }^{5,6}$ Intimal hyperplasia, which causes stenosis around the venous anastomosis, is the most prevalent access-related consequence. Thrombosis, infection, aneurysm formation, and limb 
ischemia are among the other accessrelated problems. ${ }^{7-10}$ When dialysis is required quickly, a double-lumen dialysis catheter is used to get vascular access, which is usually through the jugular or femoral vein ${ }^{7,10}$

Central lines are predominantly inserted into the neck via the internal jugular vein. They can also be inserted via the subclavian vein just below the clavicle, or, as a last resort, via the common femoral vein in the groin. ${ }^{7,10}$ They are used when short-term central access is required $(<10$ days). They come in either single-lumen or multi-lumen forms. They can be used for the administration of all intravenous solutions, including antibiotics, fluids, medications, parenteral nutrition, and chemotherapy. ${ }^{7,10}$ They are excellent for resuscitation and hemodynamic monitoring. Patients having major surgery often have them inserted in theatre. There is a range of temporary and permanent dialysis catheters for use in both acute and chronic renal failure settings. ${ }^{710}$ Again, they are inserted via the central veins under ultrasound. Long-term dialysis catheters are tunneled out onto the skin as with the previously mentioned tunneled catheters. Dialysis patients will often have such a line fitted whilst they are waiting for a more definitive form of vascular access, i.e., fistula/peritoneal dialysis line.

The 2019 KDOQI vascular access recommendations take a more patientcentered approach, acknowledging doctors' differing practice patterns while maintaining focusing on high-quality standards that provide dialysis access options tailored to each patient's goals and desires. ${ }^{6}$ The optimum access is no longer "a fistula," but rather any type of access that is dependable, capable of delivering enough dialysis without difficulties, and appropriate for each patient's needs: “The appropriate access, in the appropriate patient, at the appropriate time, for the appropriate reasons"6 The guidelines also take a fresh look at earlier issues in order to improve the patient's access options. For example, they propose a novel dialysis catheter placement sequence, prioritizing femoral catheterization over subclavian catheterization in emergency dialysis situations until an AV access or peritoneal dialysis (PD) catheter can be quickly created and used, which is justified by the potential to limit central stenosis. ${ }^{6}$

Pseudoaneurysms (PSA) usually occur due to vessel wall defects from repeated cannulations in the same location and are noted intraoperatively as having a Swiss cheese appearance. ${ }^{7,8,11}$ They can appear in AVFs, but they are more common in AVGs. True aneurysms are unlikely to arise in the prosthetic segment of an AVG, given the rigorous definition, because they need dilation of all three vessel walls. PSA can also occur at the site of anastomosis in the AVF or AVG, albeit the underlying causes are different, and include procedure-related technical flaws and infection. PSA is caused by vascular wall flaws caused by repeated cannulations in the same area and is described as having a Swiss cheese appearance intraoperatively. They can appear in AVFs, but they are more common in AVGs. True aneurysms are unlikely to arise in the prosthetic segment of an AVG, given the rigorous definition, because they need dilation of all three vessel walls. PSA can also occur at the site of anastomosis in the AVF or AVG, albeit the underlying causes are different, and include procedure-related technical flaws and infection. All patients with symptomatic AV access aneurysms or PSA should be examined and treated. ${ }^{6-8,10,11}$

A throbbing lump is the most obvious sign of a PSA.7, ${ }^{7,11}$ The borders are not so firm because these lumps are located under the strong fascial tissue. Usually, you will feel systolic vibrations throughout the lump, sometimes mistaken for an abscess or a neoplasm. Can also occur with arterial-venous fistulas. The angiographic examination is necessary when in doubt or if the location of the lesion is difficult to reach on clinical examination. ${ }^{8,10,11}$ Sonographic examination can also help to determine the size and location of this PSA. ${ }^{8,9,11}$ By locating and temporarily binding the proximal and distal arteries of this lesion, arterial reconstruction can be carried out freely. Sometimes it only takes a few lateral sutures to close this artery lesion. There is very little chance of spontaneous healing when a PSA has occurred.

The investigation of choice is duplex or Doppler ultrasound. ${ }^{7,8,11}$ This examination has a sensitivity of $94 \%$ with a specificity of
97\%. On the B-mode image, it is difficult to distinguish a PSA from a hematoma. With color Doppler, a fairly accurate picture is obtained for diagnosing PSA, which is a picture of the pulsatile flow from the blood vessels to the associated pouch. The B mode image will provide an overview of the echo lucent pocket which relaxes and contracts as the heart contracts and the channel such as the neck (neck) that drains blood from the original blood vessels to the pouch. In color Doppler, you can see a rotating flow with turbulence in the bag like a "yin yang" image. In the pulsed-wave Doppler analysis, a "to and fro" image is obtained. ${ }^{7,8,11}$ PSA can be caused by trauma, to the connection between the arterial and synthetic graft anastomoses, iatrogenic, and infection or inflammation.

\section{Anesthesia Management}

Patients with CKD are at increased risk for perioperative complications, and their general medical condition and the planned operative procedure dictate monitoring requirements. ${ }^{1,3,4,9,10}$ Blood pressure should not be taken with a cuff on an arm with an arteriovenous fistula due to the risk of thrombosis. In patients with poorly managed hypertension, continuous invasive or noninvasive blood pressure monitoring may be recommended. Debilitated or critically ill patients, as well as those who have recently undergone hemodialysis and are still somewhat hypovolemic, should have their induction agent dose lowered. ${ }^{1,3,9}$

A balanced anesthetic technique using a volatile agent, muscle relaxant, and opioids is most often employed. .,3,4,9 $^{\text {The }}$ ability to eliminate volatile anesthetics is unaffected by renal function. Potent volatile anesthetics are useful for reducing the doses of muscle relaxants required for appropriate surgical relaxation and regulating intraoperative systemic hypertension. To prevent endangering oxygen delivery to the tissues, blood flow reductions must be kept to a minimum. Opioids are beneficial since they have no cardio-depressant properties and may reduce the requirement for volatile anesthetics. In patients with CKD, however, the elimination half-life of fentanyl may be prolonged. 
Controlled ventilation should be considered for patients with kidney failure under general anesthesia. ${ }^{1,3,9}$ Under anesthesia, insufficient spontaneous breathing combined with increasing hypercarbia can cause respiratory acidosis, which can exacerbate preexisting acidemia, cause potentially severe circulatory depression, and dangerously raise serum potassium levels. Respiratory alkalosis, on the other hand, can be harmful because it moves the hemoglobin dissociation curve to the left, exacerbates hypocalcemia, and reduces cerebral blood flow.

Patients dependent on hemodialysis require special attention with respect to perioperative fluid management. ${ }^{1,3,4,9}$ An absence of renal function narrows the margin of safety between insufficient and excessive fluid administration.

\section{Post-Anesthesia Care}

Monitoring of possible complications such as infection, bleeding, and prolonged action of anesthetics. ${ }^{1,3,4,9}$ Monitoring of vital signs for possible internal bleeding and shock. Control of clinical and patient complaints such as pain or tightness. Control of surgical scars. Patients after PSA repair surgery, treated in an intensive care unit, were observed for the possibility of surgical complications.

Surgical complications, in general, are bleeding, infection, slow healing of wound repair pseudoaneurysms, tissue damage due to surgery, and prolonged effects of anesthetics. In terms of time, the early complications after surgery are bleeding, while the complications that occur late are the formation of new hematomas and infections which are common complications. ${ }^{8}$ As for infectious complications, which often occur in incisions, severe infections can occur in the stripper duct marks.

\section{CONCLUSION}

CKD is a condition characterized by a progressive and permanent decline in kidney function over a period of more than 3 months. CKD patients who need HD can be at risk of PSA due to tissue damage around arteries due to the insertion of HD vascular access. PSA requires surgery if they start to cause complaints to the patient. Anesthesia management in patients with CKD requires an understanding of the pathologic changes that accompany the renal disease, co-existing medical conditions, and the impact of reduced renal function on drug pharmacokinetics. However, the perioperative complications that arise can be managed safely if they are well anticipated and prepared perioperatively in advance. Furthermore, other case reports or case series are needed to draw more definite conclusions regarding the effects of using anesthesia management in patients with CKD.

\section{ACKNOWLEDGMENTS \\ DISCLOSURES}

\section{Funding}

This study doesn't receive any specific grant from the government or any private sector.

\section{Conflict of Interest}

The authors declare that there is no conflict of interest regarding the publication of this article.

\section{Author Contribution}

All authors contributed equally to this article

\section{Ethical Statement}

The patient has already given permission for this case report

\section{REFERENCES}

1. Holt N. Renal Disease. In: Hines RL, editor. Stoelting's: Anesthesia and Co-existing Disease 7th Edition. Philadelphia: Elsevier; 2018. p. 425-48.

2. Hastie J. Renal Physiology. In: Shafer S, editor. Stoelting's: Pharmacology \& Physiology in Anesthetic Practice 5th Edition. Philadelphia: Wolter Kluwer Health; 2015. p. 418-31.

3. Butterworth JF, Mackey DC, Wasnick JD. Anesthesia for Patients with Kidney Disease. In: Morgan \& Mikhail's Clinical Anesthesiology 6th Edition. Philadelphia: Wolter Kluwer Health; 2018. p. 631-69.

4. Dhar P, Yao FS. Kidney Transplant. In: Yao FS, editor. Yao \& Artusio's Anesthesiology: Problem Oriented Patient Management 6th Edition. Philadelphia: Lippincott Williams \& Wilkins; 2008. p. 824-47.

5. Jameson JL, Fauci AS, Kasper DL, Hauser LS, Longo DL, Loscalzo J. Nephrology. In: Harrison's Manual of Medicine 20th Edition. New York: McGraw-Hill Education; 2020. p. 711-810.

6. Lok CE, Huber TS, Lee T, Shenoy S, Yevzlin AS, Abreo K, et al. KDOQI clinical practice guideline for vascular access: 2019 update. Am J Kidney Dis. 2020;75(4):S1-164.

7. Forsyth JM, Shalan A, Thompson A. Venous Access Glossary [Internet]. Venous Access Made Easy. CRC Press; 2019. p. 3-12. Available from: http://dx.doi.org/10.1201/9780429433801-1

8. Suhardi. Vascular Trauma Management. 2017;

9. Hata MH. Preoperative Patient Assesment and Management. In: Barash P, editor. Clinical Anesthesia 7th Edition. Philadelphia: Lippincott Williams \& Wilkins; 2013. p. 583-611.

10. Putri YS, Yadi DF. Axillary Block on a Patient with Pseudoaneurism on Left Antebrachii with Terminal Renal Failure. J Anestesi Perioper. 2014;2(1):79-84.

11. Bodenham AR, Simcock L. Complications of Central Venous Access [Internet]. Central Venous Catheters. Wiley-Blackwell; p. 175-205. Available from: http://dx.doi. org/10.1002/9780470750186.ch12

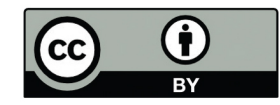

This work is licensed under a Creative Commons Attribution 